\title{
A Vanillin-Based Epoxy Vitrimer with High Performance and Closed-Loop Recyclability
}

\author{
Hafeezullah Memon ${ }^{\dagger, t}$, Haiyang Liu ${ }^{\dagger, t}$, Muhammad A. Rashid ${ }^{\dagger, t}$, Li Chen ${ }^{\dagger, t}$, Qiuran

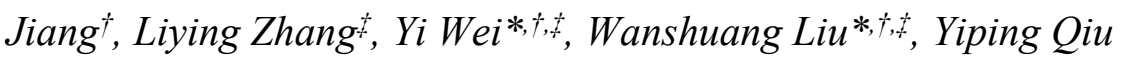

†Key Laboratory of Textile Science \&Technology, Ministry of Education, College of Textiles, Donghua University, 2999 North Renmin Road, Shanghai, 201620, China

†enter for Civil Aviation Composites, Donghua University, 2999 North Renmin Road, Shanghai, 201620, China 


\section{Degradation and chemical recycling of cured epoxy resins}

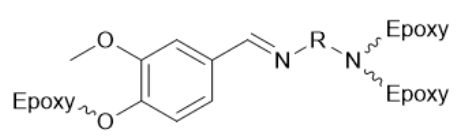

Epoxy networks

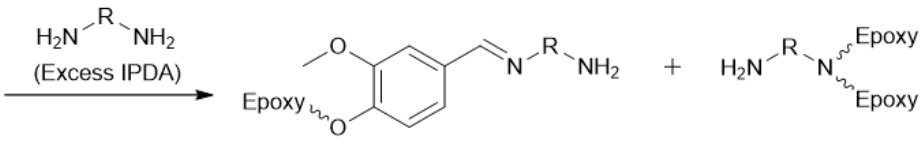

a

b

Degradation products

Scheme S1. Degradation of the cured epoxy resin (epoxy/IH-VAN) in IPDA/ethanol solution. 


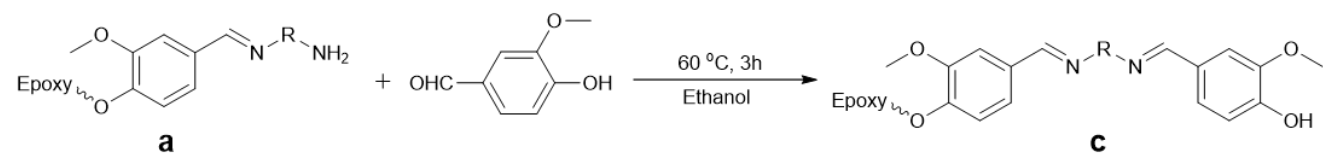

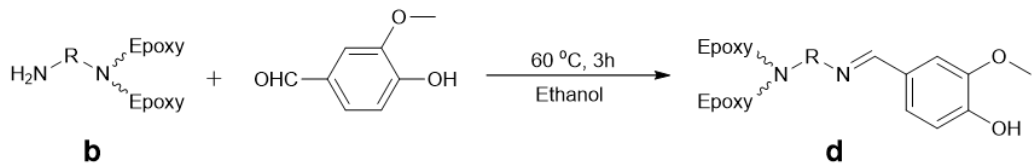

$$
\text { IPDA }
$$

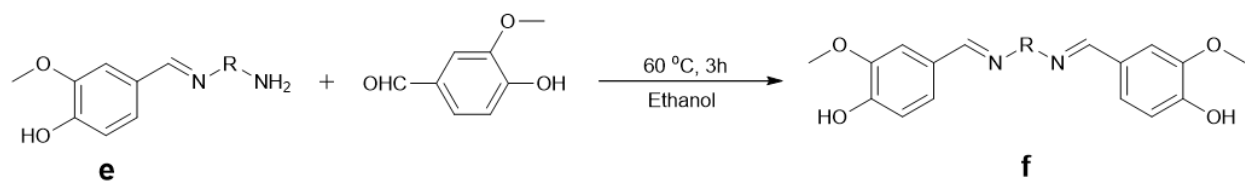

Scheme S2. The possible reactions of vanillin with the degraded epoxy resin (epoxy/IH-VAN) and residual IPDA. 


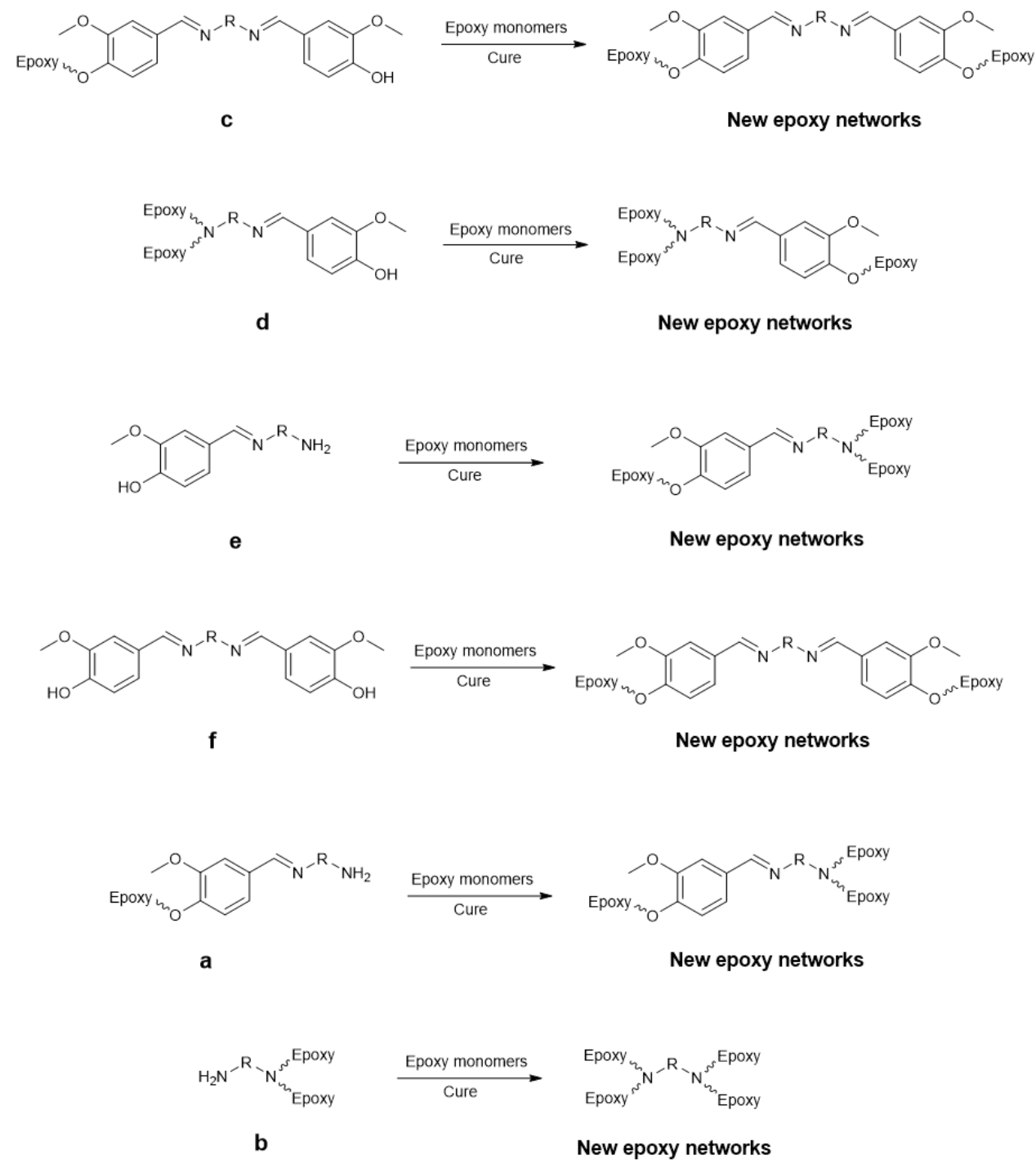

Scheme S3. The possible curing reactions for the chemically recycled epoxy resin (epoxy/IH-VAN).

Discussion: It should note that if reaction 4 in Scheme S2 occurred, there would be residual degradation products a and/or b before adding the epoxy monomers. This is because the mole ratio between IPDA (used to degrade the epoxy resin) and vanillin is 1:1. Reaction 4 in Scheme S2 might be more preferred, since degradation products a and $\mathrm{b}$ (as oligomers) have low molecular mobility, which would decrease the reactivity of their terminal amino groups. In addition, the chemical recycling process of epoxy/IHHB is similar to that of epoxy/IH-VAN. 


\section{2. ${ }^{13}$ C-NMR spectroscopy}

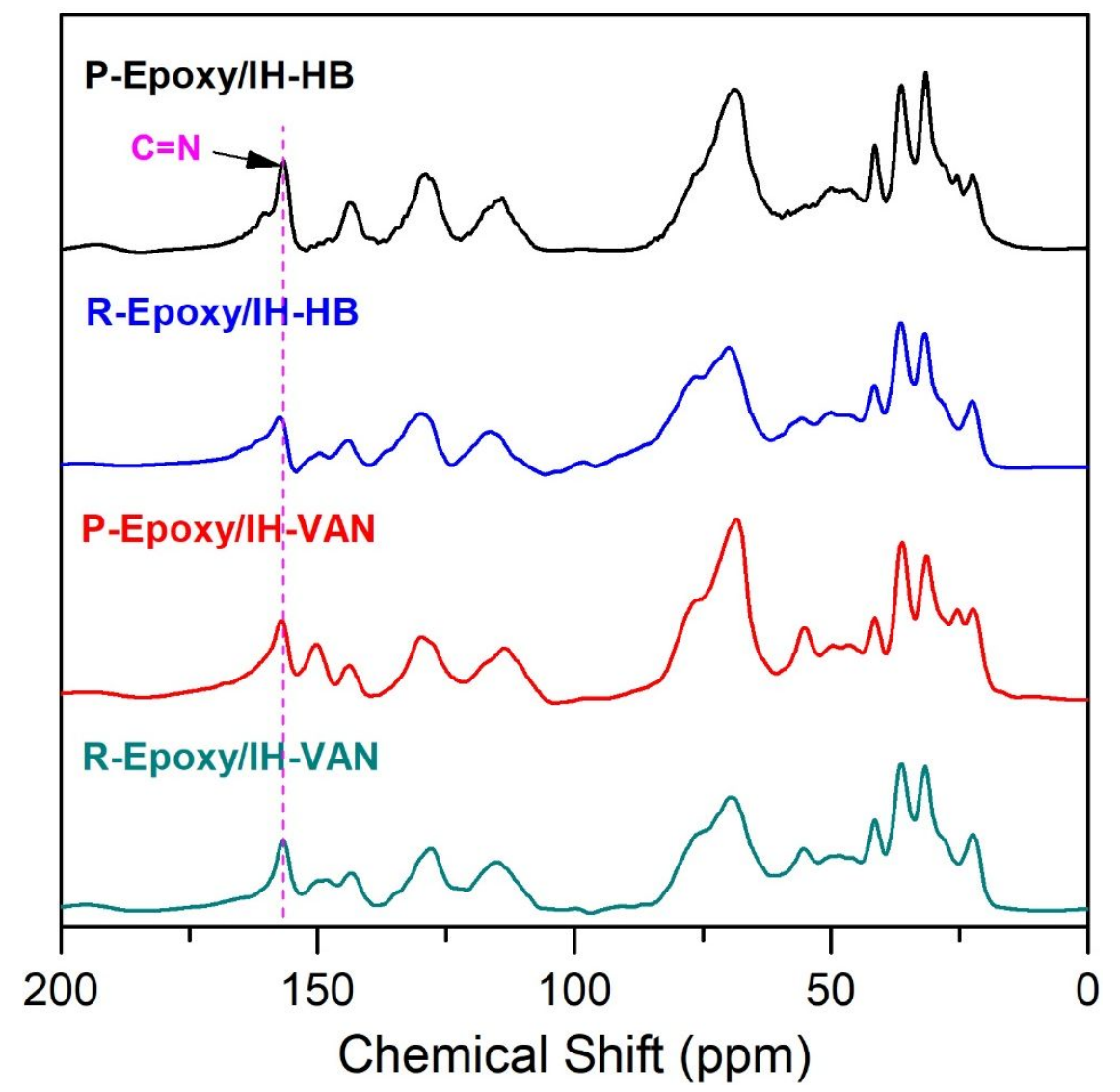

Figure S1. ${ }^{13} \mathrm{C}$ NMR spectra of pristine and chemically recycled epoxy/IH-VAN and epoxy/IH-HB resins. 


\section{Dynamic mechanical analysis}

(a)

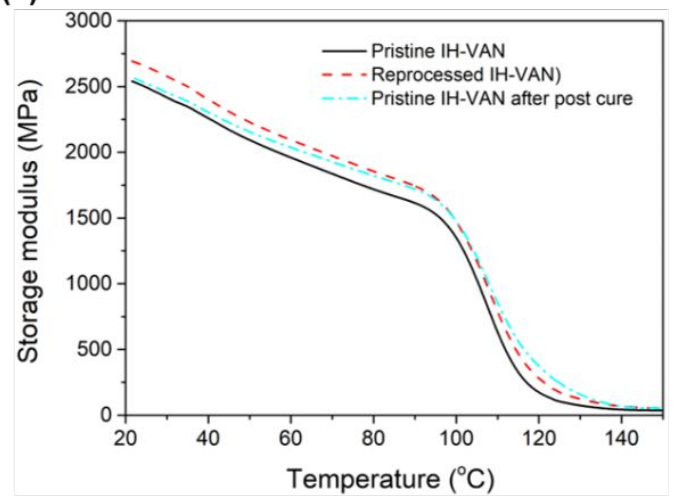

(b)

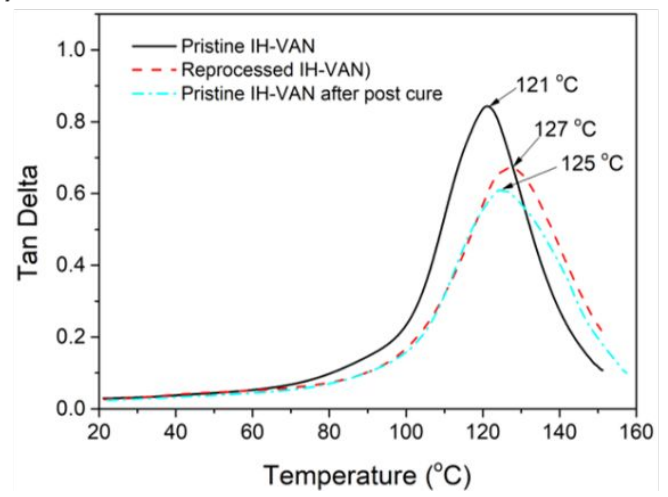

(c)

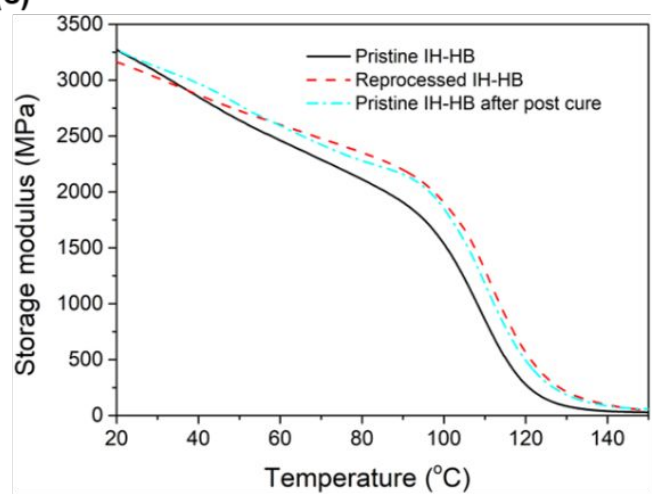

(d)

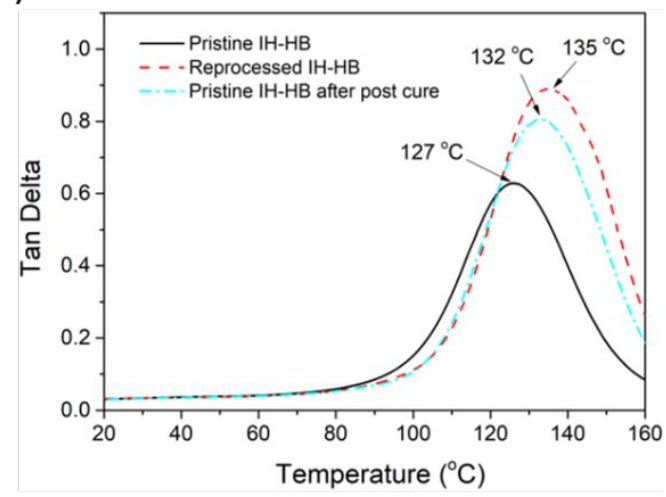

Figure S2. Storage modulus and $\tan \delta$ versus temperature curves of pristine, reprocessed and post-cured epoxy resins using IH-VAN (a-b) and IH-HB (c-d) as hardeners. 
4. Thermal gravimetric analysis

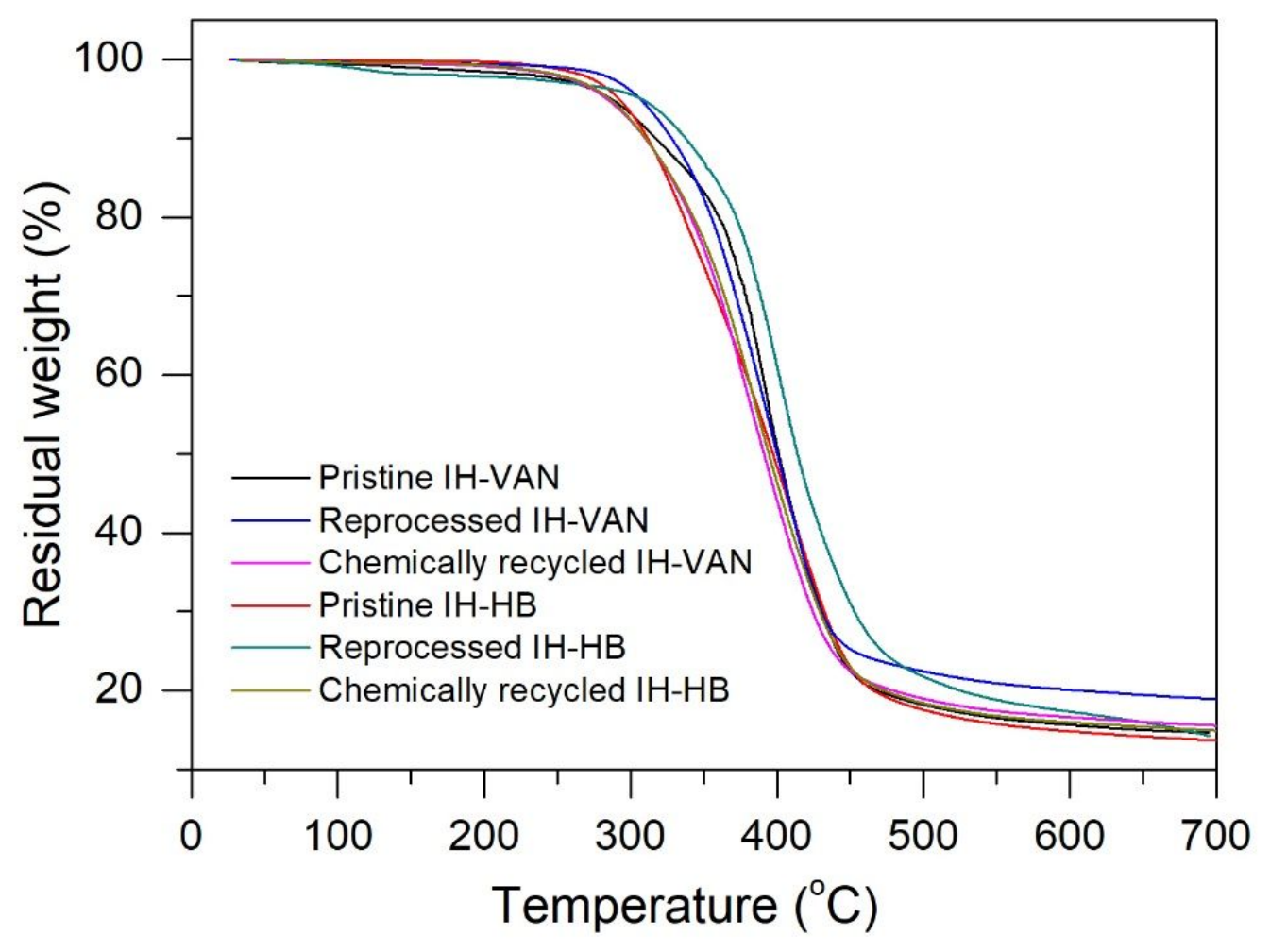

Figure S3. TGA curves of pristine and recycled epoxy resins under a nitrogen atmosphere. 


\section{Temperature variable Fourier transform infrared spectroscopy}

(a)

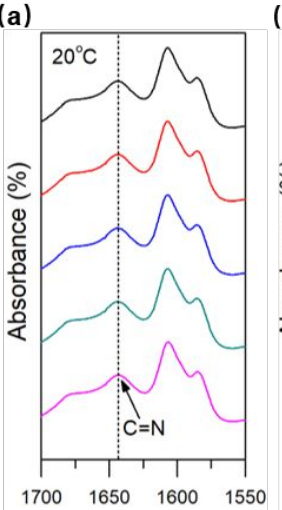

$\begin{array}{llll}1700 & 1650 & 1600 & 1550 \\ \text { Wavenumber }\left(\mathrm{cm}^{-1}\right)\end{array}$

(h)

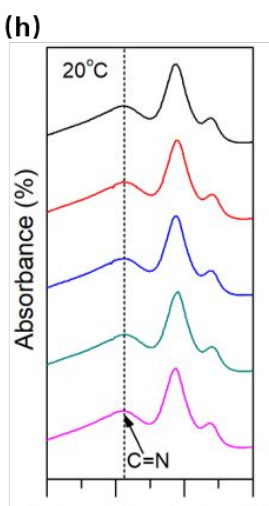

(b)

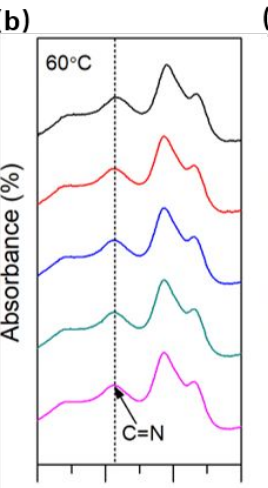

(c)

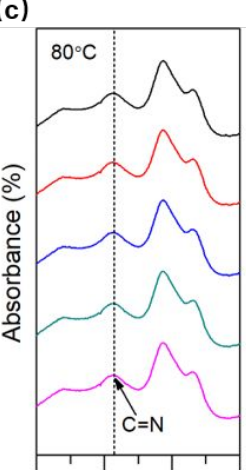

(d)

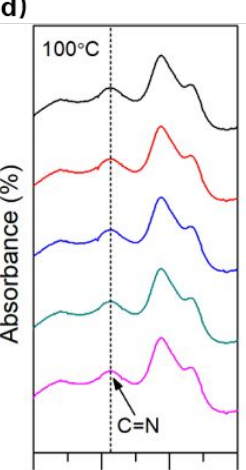

(e)

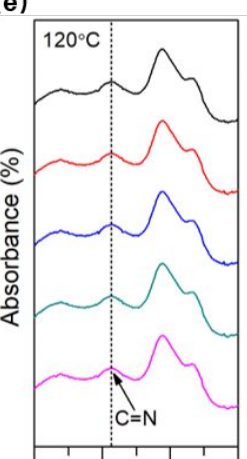

(f)
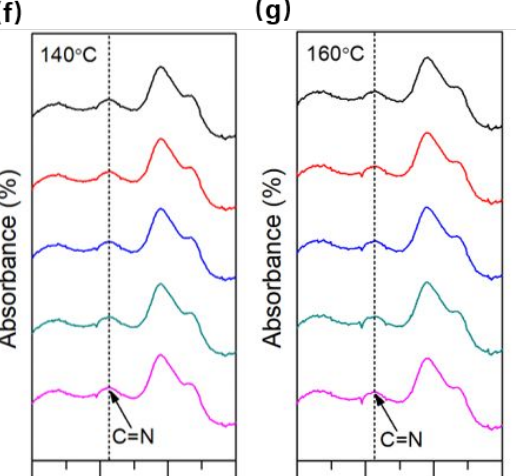

$\begin{array}{llllllll}1700 & 1650 & 1600 & 1550 & 1700 & 1650 & 1600 & 1550\end{array}$ Wavenumber $\left(\mathrm{cm}^{-1}\right) \quad$ Wavenumber $\left(\mathrm{cm}^{-1}\right) \quad$ Wavenumber $\left(\mathrm{cm}^{-1}\right) \quad$ Wavenumber $\left(\mathrm{cm}^{-1}\right) \quad$ Wavenumber $\left(\mathrm{cm}^{-1}\right)$ Wavenumber $\left(\mathrm{cm}^{-1}\right)$
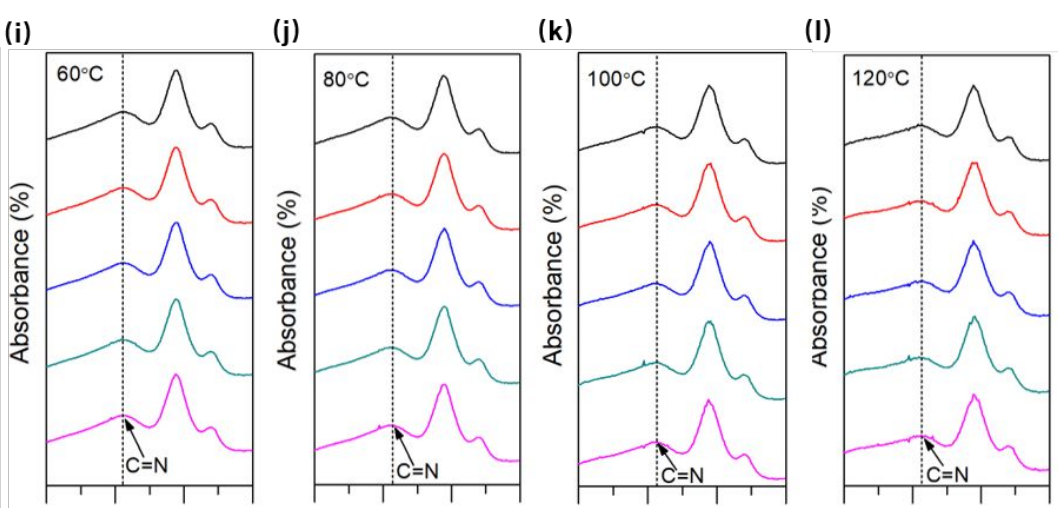

(m) $(n)$
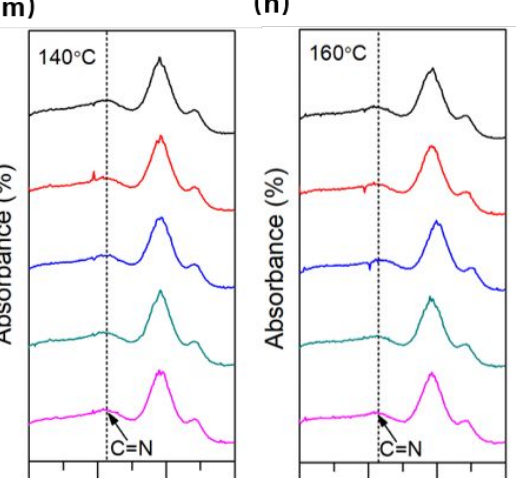

$\begin{array}{llllllllllllllllllllllllllll}1700 & 1650 & 1600 & 1550 & 1700 & 1650 & 1600 & 1550 & 1700 & 1650 & 1600 & 1550 & 1700 & 1650 & 1600 & 1550 & 1700 & 1650 & 1600 & 1550 & 1700 & 1650 & 1600 & 1550 & 1700 & 1650 & 1600 & 1550\end{array}$ Wavenumber $\left(\mathrm{cm}^{-1}\right)$ 
Figure S4. Variable temperature FTIR spectra of epoxy resins cured by IH-VAN (a-g) and IH-HB (h-n) from 20 to $160{ }^{\circ} \mathrm{C}$.

\begin{tabular}{|c|c|c|c|c|c|c|c|c|}
\hline \multirow{2}{*}{$\begin{array}{c}\text { Type of } \\
\text { dynamic bond }\end{array}$} & \multicolumn{2}{|c|}{ Pristine resins } & \multicolumn{2}{|c|}{ Reprocessed resins } & \multicolumn{3}{|c|}{ Chemically recycled resins } & \multirow{2}{*}{$\begin{array}{l}\text { Ref number } \\
\text { in the paper }\end{array}$} \\
\hline & $\begin{array}{c}\text { Tensile strength } \\
\text { (MPa) }\end{array}$ & $\begin{array}{c}T_{\mathrm{g}} \\
\left({ }^{\circ} \mathrm{C}\right)\end{array}$ & $\begin{array}{c}\text { Tensile strength } \\
\text { (MPa) }\end{array}$ & $\begin{array}{l}T_{\mathrm{g}} \\
\left({ }^{\circ} \mathrm{C}\right)\end{array}$ & $\begin{array}{c}\text { Tensile strength } \\
\text { (MPa) }\end{array}$ & $\begin{array}{c}T_{\mathrm{g}} \\
\left({ }^{\circ} \mathrm{C}\right)\end{array}$ & $\begin{array}{c}\text { Closed-loop } \\
\text { recycling }\end{array}$ & \\
\hline Siloxane & 46.6 & 83 & 47.1 & 83 & - & - & No & 20 \\
\hline Ester & 2.8 & 30 & - & - & 2.5 & 33 & Yes & 33 \\
\hline Ester & 26 & 42 & 14 & & - & 25 & Yes & 14 \\
\hline Ester & 69.2 & 187 & - & - & - & - & No & 34 \\
\hline Disulfide & 11 & 41 & 9 & 39 & - & - & No & 35 \\
\hline Disulfide & 38 & 133 & 37 & - & - & - & No & 15 \\
\hline Disulfide & 88 & 130 & 82 & 135 & - & - & No & 16 \\
\hline Imine & 46 & 71 & - & - & 41 & 72 & No & 18 \\
\hline Imine & 73 & 100 & 54 & - & - & - & No & 36 \\
\hline Imine & 81 & 172 & 80 & 175 & - & - & No & 19 \\
\hline \multirow{2}{*}{ Imine } & 60.1 (IH-VAN) & 121 & 53.6 & 127 & 60.9 & 119 & Yes & \multirow{2}{*}{ This work } \\
\hline & $60.6(\mathrm{IH}-\mathrm{HB})$ & 127 & 52.4 & 135 & 58.3 & 124 & Yes & \\
\hline
\end{tabular}


Table S1. Comparison of thermal and mechanical properties of epoxy vitrimers containing different dynamic covalent bonds. 